SICS Technical Report T2003:08 ISRN: SICS-T-2003/08-SE ISSN: 1100-3154

\title{
Wide Area Measurements of Voice Over IP Quality
}

\section{Ian Marsh and Fengyi Li}

\author{
Swedish Institute of Computer Science \\ Box 1263, SE-164 29 Kista, Sweden
}

15th May, 2003

\begin{abstract}
Time, day, location and instantaneous network conditions largely dictate the quality of Voice over IP calls. In this paper we present the results of over 18000 VoIP measurements, taken from nine sites connected in a full-mesh configuration. We measure the quality of the routes on a hourly basis by transmitting a pre-recorded call between a pair of sites. We repeat the procedure for all nine sites during the one hour interval. Based on the obtained jitter, delay and loss values as defined in RFC 1889 (RTP) we conclude that the VoIP quality is acceptable for all but one of the nine sites we tested. We also conclude that VoIP quality has improved marginally since we last conducted a similar study in 1998.
\end{abstract}




\section{Introduction}

It is well known that the users of real-time voice services are sensitive and susceptible to variable audio quality. If the quality deteriorates below an acceptable level or is too variable, users often abandon their calls and retry later. Since the Internet is increasingly being used to carry real-time voice traffic, the quality provided has become, and will remain an important issue. The aim of this work is therefore to disclose the current quality of voice communication at end-points on the Internet.

It is intended that the results of this work will be useful to many different communities involved with real-time voice communication. Within the next paragraph we list some potential groups to whom this work might have relevance. Firstly end users can determine which destinations are likely to yield sufficient quality. When deemed insufficient they can take preventative measures such as adding robustness, for example in the form of forward error correction to their conversations. Operators can use findings such as these to motivate upgrading links or adding QoS mechanisms where poor quality is being reported. Network regulators can use this kind of work to verify the quality level that was agreed upon, has indeed been deployed. Speech coder designers can utilise the data as input for a new class of codecs, of particular interest are designs which yield good quality in the case of bursty packet loss. Finally, researchers could use the data to investigate questions such as, "Is the quality of real-time audio communication on the Internet improving or deteriorating?".

The structure of the paper is as follows: Section 2 begins with some background on the quality measures we have used in this work namely, loss, delay and jitter. Following on from the quality measures, section 3 gives a description of the methodology used to ascertain the quality. In section 4 the results are presented, and due to space considerations we condense the results into one table showing the delay, loss and jitter values for the paths we measured. In section 5 the related work is given, comparing results obtained in this study with other researchers' work. This is considered important as it indicates whether quality has improved or deteriorated since those studies. Section 6 rounds off with some conclusions and a pointer to the data we have collated.

\section{What Do We Mean by Voice over IP Quality?}

Ultimately, users judge the quality of voice transmissions. Organisations such as ETSI, ITU, TIA, RCR plus many others have detailed mechanisms to assess voice quality. These organisations are primarily interested in speech coding. Assigning quality 'scores' involves replaying coded voice to both experienced and novice listeners and asking them to adjudge the perceived 
quality. Measuring the quality of voice data that has been transmitted across a wide area network is more difficult. The network inflicts its own impairment on the quality of the voice stream. By measuring the delay, jitter and loss of the incoming data stream at the receiver, we can provide some indication on how suitable the network is for real-time voice communication. The two schemes can be combined as was proposed by the ITU using with the E-model [1].

The quality of VoIP sessions can be quantified by the network delay, packet loss and packet jitter. We emphasise that these three quantities are the major contributors to the perceived quality as far as the network is concerned. The G.114 ITU standard states that the end-to-end one way delay should not exceed $150 \mathrm{~ms}$ [2]. Delays over this value adversely effect the quality of the conversation. An alternative study by Cole and Rosenbluth state that users perceive a linear degradation in the quality up to $177 \mathrm{~ms}$ [3]. Above this figure the degradation is also linear although markedly worse. As far as the packet loss is concerned, using simple speech coding such Alaw or $\mu$-law coding, tests have shown that the mean packet loss should not exceed $10 \%$ before glitches due to lost packets seriously affect the perceived quality. Note that a loss rate such as this does not say anything about the distribution of the losses. As far as the authors are aware of, no results exist that state how jitter solely can affect the quality of voice communication. Work on jitter and quality are often combined with loss or delay factors. When de-jittering mechanisms are employed, the network jitter is typically transferred into application delay. The application must hold back a sufficient number of packets in order to ensure smooth, uninterrupted playback of speech. To summarise, we refer to the quality as a combination of delay, jitter and loss. It is important to mention we explicitly do not state how these values should be combined. The ITU E-model is one approach but others exist, therefore we refer the interested reader to the references given as well as [4] and [5].

\section{Simulating and Measuring Voice over IP Ses- sions}

Our method to measure VoIP quality is to send pre-recorded calls between globally distributed sites. Through the modification of our own VoIP tool, Sicsophone, the intervening network paths are probed by a 70 second prerecorded 'test signal'. The goal of this work is therefore to report in what state the signal emerges after traversing the network paths. Incidentally, we do not include the signalling phase, i.e. establishing a connection with the remote host, rather we concentrate solely on the quality of the data (or speech) transfer.

Nine sites have been carefully chosen with large variations in hops, geo- 
graphic distances and time-zones to obtain a diverse selection of distributed sites. One important limitation of the available sites was they were all located at academic institutions, which are typically associated with well provisioned networks. Their locations are shown in the map of Figure 1. The sites were connected as a full mesh allowing us, in theory, to measure

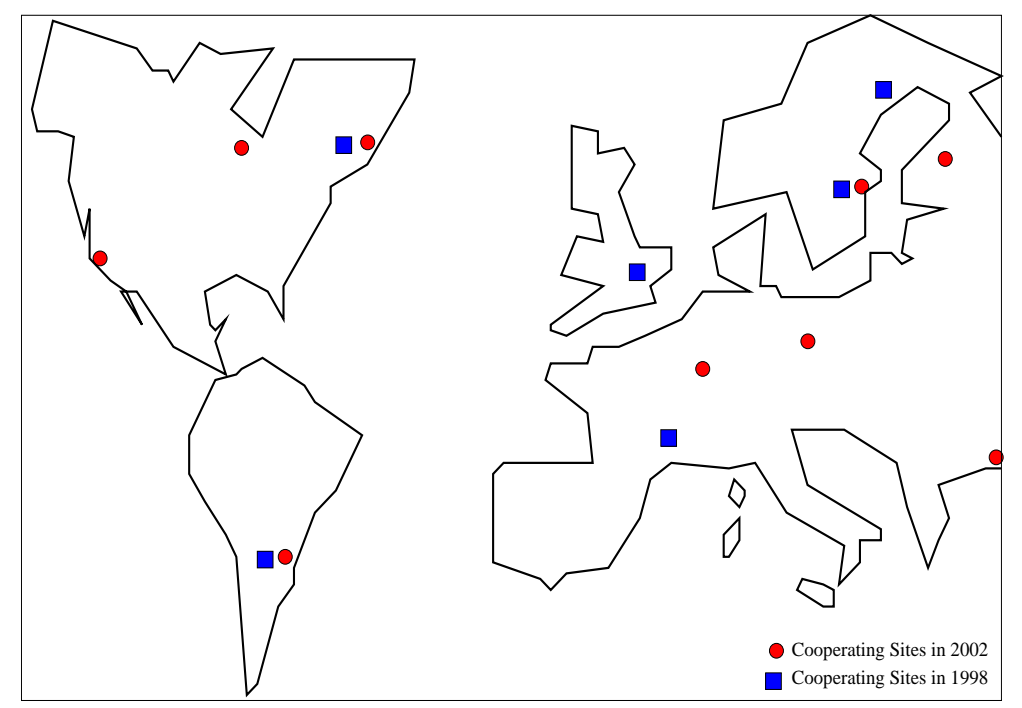

Figure 1: The nine sites used in the 2002 measurements are shown with circles. The six depicted with squares show those that were available to us in 1998, three remained unchanged during the four years.

the quality of 72 different Internet paths. In practice, some of the combinations were not usable due to certain ports being blocked, thus preventing the audio being sent to some sites. There were four such cases. Bi-directional sessions were scheduled on a hourly basis between any two given end systems. Calls were only transferred once per hour due to load considerations on remote machines.

In Table 1 below we list the characteristics of the call we used to probe the Internet paths between those sites indicated on the map. Their locations, separation in hops and time zones are given in the results section. As stated, the call is essentially a fixed length PCM coded file which can be sent between the sites, the length of the call and the payload size were arbitrarily chosen. Over a 15 week period we gathered just over 18,000 recorded sessions. The number of sessions between the nine sites is not evenly distributed due to outages at some sites, however we attempted to ensure an even number of measurements per site, in total nearly 33 million individual packets were transmitted during this work. 


\begin{tabular}{|c|c|}
\hline \multicolumn{2}{|c|}{ Test "signal" } \\
\hline Call duration & 70 seconds \\
Payload size & 160 bytes \\
Packetisation time (ms) & $20 \mathrm{~ms}$ \\
Data rate & $64 \mathrm{kbits} / \mathrm{sec}$ \\
With silence suppression & 2043 packets \\
Without silence suppression & 3653 packets \\
Coding & 8 bit PCM \\
Recorded call size & 584480 bytes \\
\hline \multicolumn{2}{|c|}{ Obtained data } \\
\hline Number of hosts used (2003) & 9 \\
Number of traces obtained & 18054 \\
Number of data packets & $32,771,021$ \\
Total data size (compressed) & 411 Megabytes \\
Measurement duration & 15 weeks \\
\hline
\end{tabular}

Table 1: The top half of the table gives details of the call used to measure the quality of links between the sites. The lower half provides information about the data which was gathered.

\subsection{A Networking Definition of Delay}

We refer to the delay as the one way network delay. One way delay is important in voice communication, particularly if it is not the same in each direction. Measuring the one way delay of network connections without synchronised clocks is a non-trivial task. Hence many methods rely on round-trip measurements and halve the values, hence estimating the one way delay. We measured the network delay using the RTCP protocol which is part of the RTP standard [6]. A brief description follows. At given intervals the sender transmits a so called "report" containing the time the report was sent. On reception of this report the receiver records the current time. Therefore two times are recorded within the report. When returning the report to the sender, the receiver subtracts the time it initially put in the report, therefore accounting for the time it held the report. Using this information the sender can calculate the round-trip delay and importantly, discount the time spent processing the reports at the receiver. This can be done in both directions to see if any significant anomalies exist. We quote the network delay in the results section as they explicitly do not include any contribution from the end hosts. Therefore it is important to state the delay is not the end-to-end delay but the network delay. We chose not to include the delay contributed by the end system as it varies widely from operating system to operating system and how the VoIP application itself is implemented. The delay incurred by an end system can vary from $20 \mathrm{~ms}$ 
up to $1000 \mathrm{~ms}$, irrespective of the stream characteristics.

\subsection{Jitter - An IETF Definition}

Jitter is the statistical variance of the packet interarrival time. The IETF in RFC 1889 define the jitter to be the mean deviation (the smoothed absolute value) of the packet spacing change between the sender and the receiver [6]. Sicsophone sends packets of identical size at constant intervals which implies that $S_{j}-S_{i}$ (the sending times of two consecutive packets) is constant. The difference of the packet spacing, denoted $D$, is used to calculate the interarrival jitter. According to the RFC, the interarrival jitter should be calculated continuously as each packet $i$ is received. For one particular packet the interarrival jitter $J_{i-1}$ for the previous packet $i-1$ is calculated thus:

$$
J_{i}=J_{i-1}+\left(|D(i-1, i)|-J_{i-1}\right) / 16
$$

According to the RFC "the gain parameter 1/16 gives a good noise reduction ratio while maintaining a reasonable rate of convergence". As stated earlier, buffering due to jitter adds to the delay of the application. This delay is accounted for in the results we present. The "real" time needed for de-jittering depends on how the original time spacing of the packets should be restored. For example if a single packet buffer is employed it would result in an extra 20ms (the packetisation time) being added to the total delay. Note that packets arriving with a spacing greater than $20 \mathrm{~ms}$ should be discarded by the application as being too late for replay. Multiples of $20 \mathrm{~ms}$ can thus be allocated for every packet held before playout in this simple example. To summarise, the delay due to de-jittering the arriving stream is implementation dependent, thus do not include it in our results.

\subsection{Counting Ones Losses in the Network}

We calculate the lost packets as is exactly defined in RFC 1889. It defines the number of lost packets as the expected number of packets subtracted by the number actually received. The loss is calculated using expected values so as to allow more significance for the number of packets received. For example 20 lost packets from 100 packets has a higher significance than 1 lost from 5. For simple measures the percentage of lost packets from the total number of packets expected is stated. As stated the losses in this work do not include those incurred by late arrivals, as knowledge of the buffer playout algorithm is needed, therefore our values are only the network loss. Detailed analysis of the loss patterns is not given in the results section, we simply state the percentages of single, double and triplicate losses. 


\section{Results}

The results of 15 weeks of measurements are condensed into figure 2 . The table should be interpreted as an 11 by 11 matrix. The locations listed horizontally across the top of the table are the locations used as receivers. Listed vertically they are configured as senders. The values in the rightmost column and bottom row are the statistical means for all the connections from the host in the same row and to the host in the same column respectively. For example the last column of the first row (directly under "Mean") the average delay to all destinations from Massachusetts is $112.8 \mathrm{~ms}$.

Each cell includes the delay, jitter, loss, number of hops and the time difference prefixed by the letters $\mathrm{D}, \mathrm{J}, \mathrm{L}, \mathrm{H}$ and $\mathrm{T}$ for each of the connections. The units for each quantity are the delay in milliseconds, the jitter in milliseconds, the loss in percentage, the hops as reported by traceroute and time differences in hours. A ' + ' indicates that the local time from a site is ahead of the one in the corresponding cell and behind for a '-'. The values in parenthesis are the standard deviations. A NA signifies "Not Available" for this particular combination of hosts. The bottom rightmost cell contains the mean for all 18054 calls made, both to and from all the nine hosts involved.

The most general observation is the quality of the paths is generally good. The average delay is just below the ITU's G.114 recommendation for the end-to-end delay. Nevertheless at $136 \mathrm{~ms}$ it does not leave much time for the end systems to encode/decode and replay the voice stream. A small buffer would absorb the $4.1 \mathrm{~ms}$ jitter and a loss rate of $1.8 \%$ is more than acceptable with PCM coding [4].

There are two clear groupings from these results, those within the EU and the US and those outside. The connections in Europe and the United States (and between them) are very good. The average delay between the US/EU hosts is $105 \mathrm{~ms}$, the jitter is $3.76 \mathrm{~ms}$ and the loss $1.16 \%$. Those outside fair less well. The Turkish site suffers from large delays, which is not surprising as the Turkish research network is connected via a satellite link to Belgium (using the Geant network). The jitter and loss figures however are low, $5.7 \mathrm{~ms}$ and $4 \%$ respectively. The Argentinian site suffers from asymmetry problems. The quality when sending data to it is significantly worse than when receiving data from it. The delay is $1 / 3$ higher, the jitter is more than twice it in the opposite direction and the loss is nearly four times higher than when sending to it. Unfortunately we could not perform a traceroute from the host in Buenos Aires, so we cannot say how the route contributed to these values.

We now turn our attention to results which are not related to any particular site. As far as loss is concerned the majority of losses are single losses. $78 \%$ of all the losses counted in all trace files were single losses whereas $13 \%$ were duplicate losses and $4 \%$ triplicate losses. Generally the jitter is low relative to the delay of the link, approximately $3-4 \%$. This is not totally 
unexpected as the loss rates are also low. With the exception of the Argentinian site, the sites did not exhibit large differences in asymmetry and were normally within $5 \%$ of each other in each direction. It is interesting to note that the number of hops could vary under the 15 week measurement period denoted by ( $)$ in the hops field. Only very few $(<0.001 \%)$ out of sequence packets were observed. Within [7] there are details of other tests, such as the effect of using silence suppression, differing payload sizes and daytime effects. In summary no significant differences were observed in these tests. We can attribute this (and the good quality results) to generally well-provisioned academic networks.

\section{Related Work}

Similar but less extensive measurements were performed in 1998 [8]. Only three of the hosts remain from four years ago so comparisons can only be made for these routes. An improvement, in the order of $5-10 \%$ has been observed for these routes. We should point out though, the number of sessions recorded four years ago numbered only tens per host, whereas on this occasion we performed hundreds of calls from each host. Bolot et. al. looked at consecutive loss for designing an FEC scheme [9]. They concluded that the number of consecutive losses is quite low and stated that most losses are one to five losses at $8 \mathrm{am}$ and between one to ten at $4 \mathrm{pm}$. This is in broad agreement with the findings in this work, however we did not investigate the times during the day of the losses. Maxemchuk and Lo measured both

loss and delay variation for intra-state connections within the USA and international links [10]. Their conclusion was the quality depends on the length of the connection and the time of day. We did not try different connection durations but saw much smaller variations (almost negligible) during a 24 hour cycle (see [7]). We attribute this to the small 64kbits per second VoIP session on well dimensioned academic networks. It is worthy to point out our loss rates were considerably less than Maxemchuks (3-4\%). Dong Lin had similar conclusions [11], stating that in fact even calls within the USA could suffer from large jitter delays. Her results on packet loss also agree with those in [9], which is interesting, as the measurements were taken some four years later.

\section{Conclusions}

We have presented the results of 15 weeks of voice over IP measurements consisting of over 18000 recorded VoIP sessions. We conclude that the quality of VoIP is good, and in most cases is over the requirements of many speech quality recommendations. Recall that all of the sites were at academic institutions which is an important factor when interpreting these results as most 
universities have well provisioned links, especially to other academic sites. Nevertheless, the loss, delay and jitter values are very low and from previous measurements the quality trend is improving. We can only attribute this to more capacity and better managed networks than those four years ago. However some caution should be expressed as the sample period was only 15 weeks, the bandwidth of the flows very small and only used once per hour. We have a large number of sample sessions so can be confident the findings are representative of the state of the network at this time. One conclusion is that VoIP is obviously dependent on the IP network infra-structure and not only on the geographic distance. This can be clearly seen in the differences between the Argentinian and Turkish hosts. Concerning the actual measurement methodology, we have found performing measurements on this scale is not an easy task. Different access mechanisms, firewalls, NATs and not having permissions on all machines, complicates the work in obtaining (and validating later) the measurements. Since it is not possible to envisage all the possible uses for this data we have made it available for further investigation at http://www.sics.se/ ianm/COST263/cost263.html.

\section{References}

[1] ITU-T Recommendation G.107, "The E-Model, a computational model for use in transmission planning," December 1998.

[2] I.-T. Recommendation G.114, "General Characteristics of International Telephone Connections and International Telephone Circuits: OneWay Transmission Time," Feb. 1998.

[3] R. Cole and J. Rosenbluth, "Voice over IP Performance Monitoring," ACM Computer Communication Review, 2002.

[4] B. L.F.Sun, G.Wade and E.C.Ifeachor, "Impact of Packet Loss Location on Perceived Speech Quality," in Proceedings of 2nd IP-Telephony Workshop (IPTEL '01), (Columbia University, New York), pp. 114122, April 2001.

[5] N. Kitawaki, T. Kurita, and K. Itoh, "Effects of Delay on Speech Quality," NTT Review, vol. 3, pp. 88-94, Sept. 1991.

[6] H. Schulzrinne, S. Casner, R. Frederick, and V. Jacobson, "RTP: A Transport Protocol for Real-Time Applications," RFC 1889, Internet Engineering Task Force, Jan. 1996.

http://www.rfc-editor.org/rfc/rfc1889.txt.

[7] F. Li, "Measurements of Voice over IP Quality," Master's thesis, KTH, Royal Institute of Technology, Sweden, 2002. 
Wide Area Measurements of Voice Over IP Quality

[8] O. Hagsand, K. Hansson, and I. Marsh, "Measuring Internet Telephone Quality: Where are we today?," in Proceedings of the IEEE Conference on Global Communications (GLOBECOM), (Rio, Brazil), IEEE, Nov. 1999.

[9] J. Bolot, H. Crepin, and A. Garcia, "Analysis of audio packet loss in the internet," in Proc. International Workshop on Network and Operating System Support for Digital Audio and Video (NOSSDAV), Lecture Notes in Computer Science, (Durham, New Hampshire), pp. 163-174, Springer, Apr. 1995.

[10] N. F. Maxemchuk and S. Lo, "Measurement and interpretation of voice traffic on the Internet," in Conference Record of the International Conference on Communications (ICC), (Montreal, Canada), June 1997.

[11] D. Lin, "Real-time voice transmissions over the Internet," Master's thesis, Univ. of Illinois at Urbana-Champaign, 1999. 


\begin{tabular}{|c|c|c|c|c|c|c|c|c|c|c|}
\hline$\sum_{\Sigma}^{\varpi}$ & 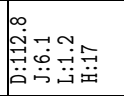 & $\mid$ & $\underset{\sim}{\mathbb{N}}$ & & 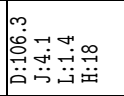 & $\begin{array}{l}\stackrel{2}{\dddot{x}} \\
\end{array}$ & 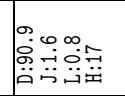 & 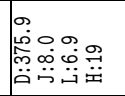 & : & \\
\hline 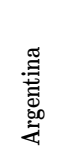 & 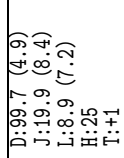 & 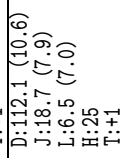 & 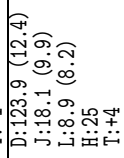 & 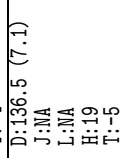 & 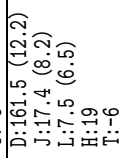 & 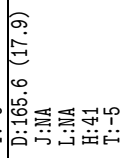 & 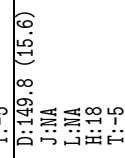 & 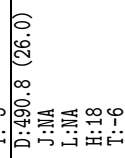 & P. & ז) \\
\hline 胥 & 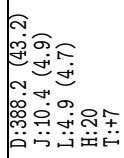 & 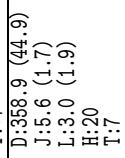 & 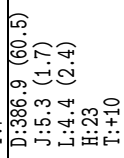 & 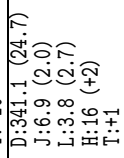 & 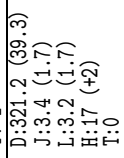 & 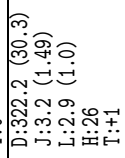 & 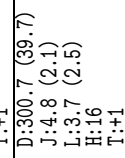 & 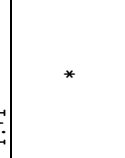 & 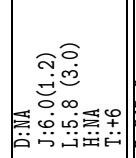 & 8 \\
\hline 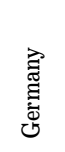 & 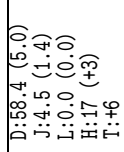 & 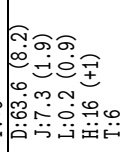 & 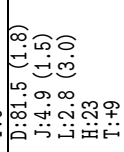 & 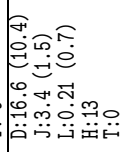 & 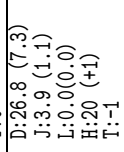 & 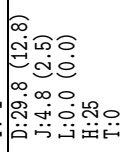 & * & 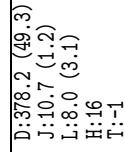 & 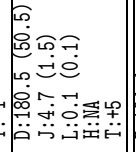 & تص \\
\hline 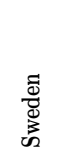 & 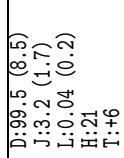 & 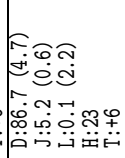 & 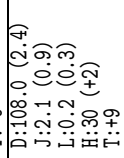 & 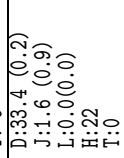 & 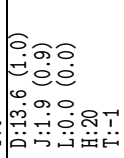 & * & 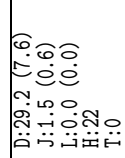 & 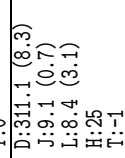 & 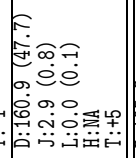 & \\
\hline 呇 & 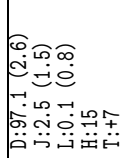 & 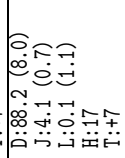 & 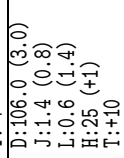 & 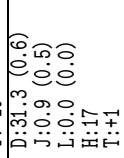 & * & 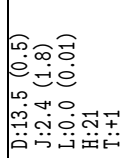 & 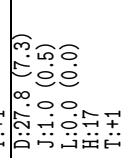 & 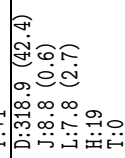 & 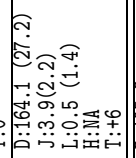 & \\
\hline 壹 & 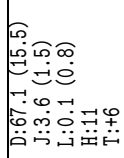 & 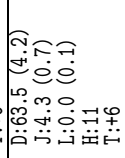 & 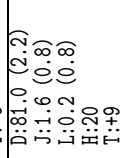 & * & 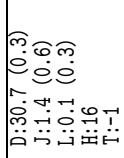 & 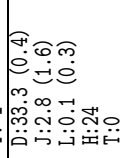 & 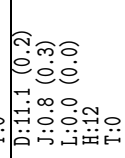 & 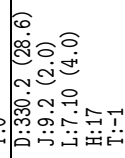 & 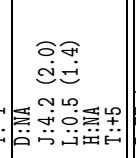 & $\ddot{\theta}$ \\
\hline & 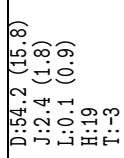 & 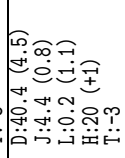 & * & 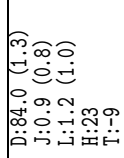 & 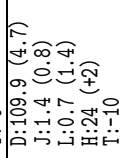 & 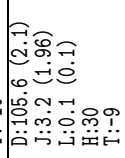 & 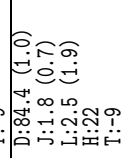 & 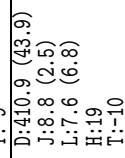 & 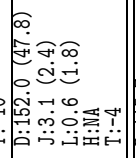 & m. \\
\hline 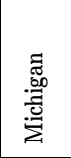 & 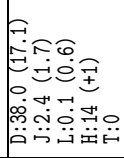 & * & 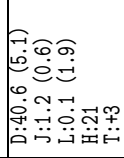 & 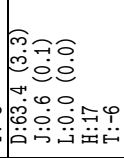 & 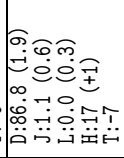 & 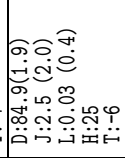 & 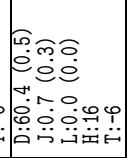 & 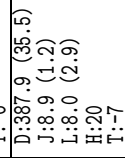 & 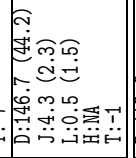 & $\mid$ \\
\hline 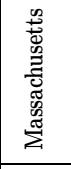 & * & 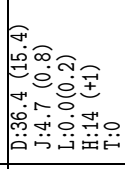 & 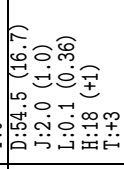 & 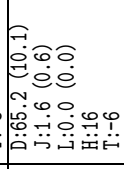 & 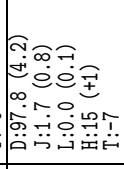 & 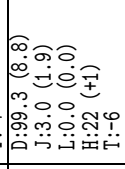 & 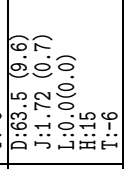 & 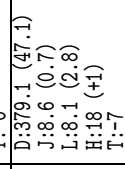 & 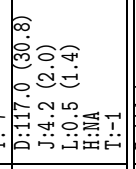 & 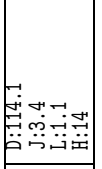 \\
\hline 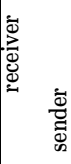 & 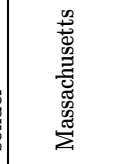 & 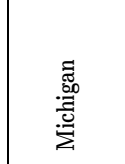 & 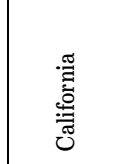 & 壱 & 蛋 & 离 & 芯 & 密 & 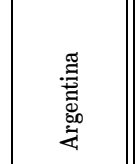 & 尔 \\
\hline
\end{tabular}

Figure 2: A summary of 18000 VoIP sessions. The delay, jitter and loss for the nine sites. The delay and jitter are in milliseconds, the losses are in percentages. The number of hops and time zones (in hours) are also given. The means for each site and all sites are stated and standard deviations are in parenthesis. 